\title{
Step response method for transmission line parameter estimation
}

\begin{abstract}
In this paper a method is proposed to determine parameters for circuits of known structure through the use of step responses. The method is developed in the frequency as well as time domains based on nonlinear minimum least squares extraction to determine the unknown parameters. Pspice circuit simulations and MATLAB simulations are performed on circuits constructed from passive elements of transmission lines, these being resistors, inductors and capacitors, to validate the method. Additionally, comparison in terms of accuracy is made with the direct method which uses time specific output data points.
\end{abstract}

Keywords: transmission lines, nonlinear least mean square, system identification, circuit parameter estimation, step response methods.
Volume 7 Issue I - 202 |

\section{Louiza Sellami}

Department of Electrical and Computer Engineering, US Naval Academy, USA

Correspondence: Louiza Sellami, Department of Electrical and Computer Engineering, US Naval Academy, Annapolis, Maryland, USA, Email sellami@usna.edu

Received: December 29, 2020 | Published: January 29, 2021

\section{Introduction}

Applications of analog circuits have increased dramatically over the years, prompting an increased interest in their design and testing. Because of their complexities, the diverse design styles and response parameters of these circuits, testing can be difficult and expensive, not to mention time-consuming. Consequently it is important to develop numerical methods for parameter estimation and, thus, detection of component failure for these circuits. Many such model based methods for specific systems already exists. ${ }^{1-4}$ In general, parameter estimation may include DC or AC specifications. For linear circuits, for instance, such as transmission lines used in communication systems, parameters include the resistance, inductance and capacitance. In order to use off the shelf systems, it is sometimes important to know how close the internal components are to the desired design values. Deviations can occur due to circuit parameter drifting, manufacturing process variations, ageing, and applications in non-intended use. This is particularly important, for instance, in transmission systems for picture processing where different transmission lines used by different manufacturers with the result that different transmission rates occur. Consequently, if one knows the actual values of the system parameters, one can design and implement compensators that would produce equal performance. Furthermore, this is very useful for establishing highest possible transmission rates. Time domain step and pulse response based methods are most commonly used for system identification, especially in process industries. Their applications to control systems, ${ }^{5}$ and synchronous machines have been documented in the literature. ${ }^{6}$ In the case of some analog circuits these techniques have been used for the purpose of fault diagnosis and testing. ${ }^{7-9}$

In this paper we propose using the alternative method of nonlinear least square fitting (NLSF) to extract the parameters of a transmission line circuit model from collected step response datasets. We show that the direct method is sensitive to the accuracy of the measurements from collected datasets, whereas the NLSF method has much lower sensitivity. The parameters are extracted from Pspice simulated responses as well as MATLAB calculations. PSspice is used to simulate the circuit in order to obtain the time domain response to the unit step. MATLAB simulations are used to calculate the parameters via NLSF and to solve nonlinear equations for the direct method. The paper is organized as follows. In the second section we present the general principle for circuit parameter extraction, and lay out the methodology for extracting the parameters. In the third section we apply the methodology to a standard linear transmission model. Section four is devoted to Pspice and MATLAB simulation results, and finally in the conclusion, we discuss the significance of these results, as well as the use of this same method in circuits containing transistors, in particular traveling wave transistors.

\section{General methodology}

Given a circuit of known configuration, which could be constructed from active and /or passive components, it is desired to determine the values of these components by making measurements at the terminals. These measurements correspond to time domain voltage responses to input voltage pulses of varying amplitudes and duty cycles, or input step. A typical schematic is shown in Figure 1. With the circuit at hand, we can symbolically evaluate responses to known excitations in terms of circuit element values taken as parameters in the time and frequency domains. Then we fit the measurements made with these excitations on the actual circuit with the symbolically determined ones by variation of the parameters. This then becomes a problem of estimation for which different criteria for best fit can be used. Due to their practical importance, we concentrate upon step and pulse inputs and measure the outputs for different values of pulse parameters, such as pulse width, amplitude and frequency.

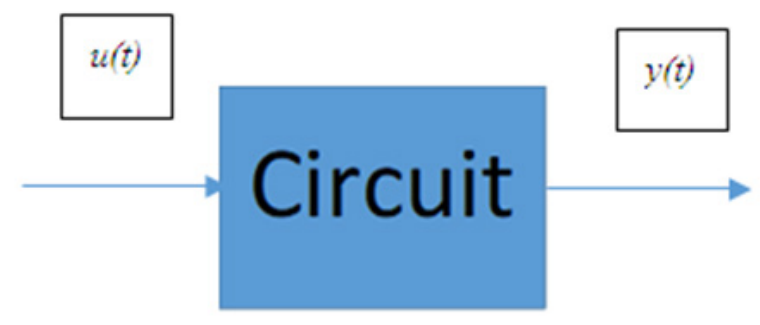

Figure I Schematic of system of interest.

In some specific applications, circuit parameters such as loop length, resistance per unit length, inductance per unit length, and capacitance also per unit length might be considered. These parameters are of great importance, since for instance they are known to limit the bandwidth and highest data transmission available to picture transmission on the line. Because these parameters vary from one connection to the other, in order to estimate them, a pulse excitation is applied to the input terminal of the line while the signal at the output 
terminal is sampled. From the sampled values, and by means of least square method, these parameters can be found.

In general, we assume the sampled circuit output vector functions are given in the form of

$$
y(t)=F(u(t, a, w, f), p)
$$

Where $p$ is the n-dimensional parameter vector, and $u(t, a, w, f)$ represents the sampled pulse input vector, with $a$ chosen amplitude $a$, width $w$ and frequency $f$. Both the input and output are functions of continuous time $t$, but are sampled at times $t j=1,2, \ldots, m \mathrm{n}$, for setting up a minimization problem for determination of the parameters. This is accomplished by taking the measured output vector $y_{\text {measured }}(t)$ and comparing it to $y(\mathrm{t})$ at the sampled time $t=t j$. Finally by using the least mean square method to minimize (2) One can solve for the unknown parameters.

$$
\sum_{j=1}^{j=m n}\left\|y_{\text {measured }}\left(t_{j}\right)-y\left(t_{j}\right)\right\|^{2}
$$

\section{Case of a lossless transmission line model}

A lossless transmission line can be represented by the circuit model show in Figure 2. The parameters are the series inductances $L_{1}$ and $L_{2}$, and the shunt capacitance $C$. The other components in the circuit are the input resistance $R_{\text {in }}$ of the voltage source, and the load resistance $R L$, which are known. To characterize this transmission line requires the determination of the three parameters $L_{1}, L_{2}$, and $C$. The input and output voltages are $V_{\text {in }}$ and $V_{\text {out }}$, with the former being a unit step function, and the latter measured across the load resistance. In our case, and as a first step, we will focus on estimating the capacitance, assuming all other parameters are known. This is because capacitance is the one parameter that is most likely to change due to parasitic effects. Furthermore, signal transmission generally has voltages changing rapidly and is thus limited in speed by capacitive effects for internal connections. In order to be able to obtain the circuit parameters, we first obtain the voltage transfer function using voltage divider rule (eq. 3):

$$
\frac{V_{\text {out }}}{V_{\text {in }}}=\frac{\frac{1}{C}}{s\left(s-p_{1}\right)\left(s-p_{2}\right)\left(s-p_{3}\right)}
$$

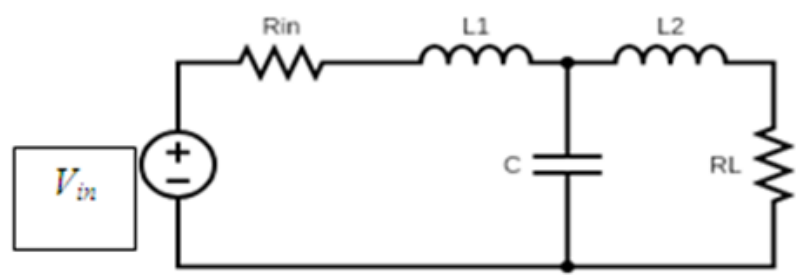

Figure 2 Circuit schematic of a lossless transmission line.

Where $p 1, p 2$, and $p 3$ are the poles which are given by

$$
\begin{aligned}
p_{1}=-1, \quad p_{2} & =-\frac{1}{2}-\frac{1}{2} \sqrt{1-\frac{8}{C}} \\
p_{3} & =-\frac{1}{2}+\frac{1}{2} \sqrt{1-\frac{8}{C}}
\end{aligned}
$$

Note in (4) the last two poles depend on the unknown parameter $C$. Further, the term under the radical gives rise to three cases.

I. Case 1: $C=8$ gives rise to two real double poles $p_{1}=p_{2}=-1 / 2$.

II. Case 2: $0<C<8$ gives rise to two complex conjugate poles per (4).

III. Case 3: $\mathrm{C}>8$ gives rise to two distinct real poles.

Since it is the time domain response that is measured, it is necessary to obtain an expression for vout $(t)$. This is done via (3) by using partial fraction expansion for a unit step input. This gives:

\section{Simulation results}

Pspice simulations for the circuit of Figure 2 were conducted for a unit step input voltage for three values of the parameter $C$ and the plots for the output voltage are shown in Figure 3. To estimate this parameter $\mathrm{C}$ from the symbolic output of equation (5), we proceed via the basic method and the nonlinear square fitting method (NLSF) respectively. The basic method consists of solving the nonlinear equation (5) in $a$ at various instants in time using MATLAB. In contrast the NLSF method consists of minimizing the error function given in (2) using all of the data points of both the measured and the symbolic output voltages, also using MATLAB. The results are shown in Table 1 for the basic method and Table 2 for the NLSF method. Based on the results of Table 1, it is clear that the estimated value of $\mathrm{C}$ changes with the time sample used. Further, the relative variation from the actual value is between $15 \%$ and $30 \%$, which are quite high and are un-desirable for real life applications. The limitation of the basic method is overcome by the NLSF method, which give noticeably better results. These are summarized in Table 2.

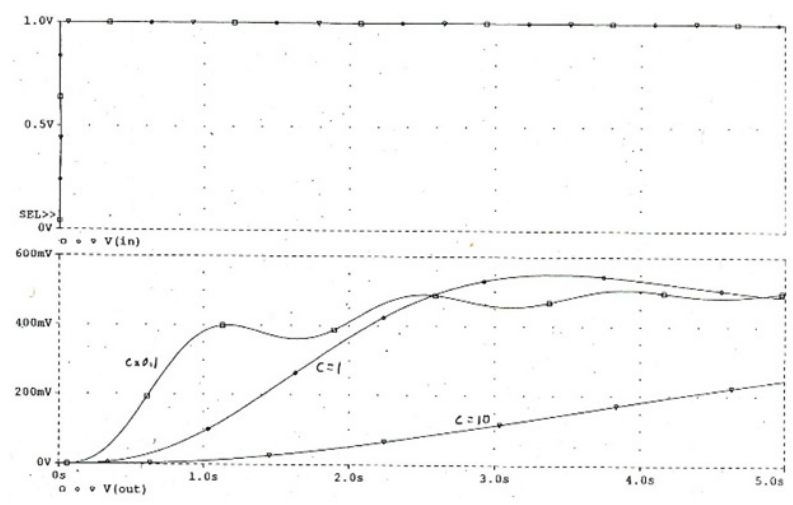

Figure 3 Output voltage for a unit step input and for three values of the parameter $\mathrm{C}$.

Table I Estimation of $\mathrm{C}$ using the basic method for the nominal value of I

\begin{tabular}{lllll}
\hline Time & Is & 2s & 3s & 4s \\
\hline Cactual & $\mathrm{I}$ & $\mathrm{I}$ & $\mathrm{I}$ & $\mathrm{I}$ \\
Cestimated & $\mathrm{I} .3$ & $\mathrm{I} .2$ & 83 & 85 \\
Relative error & $30 \%$ & $20 \%$ & $17 \%$ & $15 \%$
\end{tabular}

Table 2 Estimation of C using the NLSF method for the nominal values of $0 . I$ and $I$

\begin{tabular}{lll}
\hline$C_{\text {actual }}$ & 0.1 & $\mathrm{I}$ \\
$\mathrm{C}_{\text {estimated }}$ & 0.093 & $1.04 \mathrm{I}$ \\
Relative error & $7 \%$ & $4.1 \%$ \\
\hline
\end{tabular}




\section{Conclusion}

By using step response in the time and frequency domains, we are able to set up estimation equations via the basic method and nonlinear best fit methods. The simulation results via Pspice and MATLAB prove the supremacy of the NLSF method over the basic method. As an important application, this work could be extended to describe the active analog circuits containing transistors such as line voltages in the gate and drain of nonlinear traveling-wave field effect transistors. These special transistors, whose electrodes behave as a nonlinear transmission line, are used to amplify soliton like pulses, ${ }^{10}$ hereby compensating for the wave attenuation that would normally occur in a lumped transmission line.

\section{Acknowledgments}

None.

\section{Funding}

None.

\section{Conflicts of interest}

The authors declare that there is no conflict of interest.

\section{References}

1. Richardson AMD, Bruls EMJG, Harvey RJA, et al. Analogue Fault Simulation Based on Layout Dependent Fault Model. Proceedings of the International Test Conference. 1994:641-649.
2. Sebeke C, Teixeira JP, Ohletz MJ. Automatic Fualt Extraction and Simulation of Layout Realistic Faults for Integrated Analog Circuits. Proceedings of the European Design and Test Conference. 1995:464468.

3. Hamid NB, Kaminska B. Analog Circuit Testing Based on Sensitivity Computation and New Circuit Modeling.

4. Nagi N, Chatterjee A, Abraham JA. DRAFTS: Discretized Analog Circuit Fault Simulator. Proceedings of the 30th ACM/IEEE Design and Automation Conference. 1993:509-514.

5. Li SY, Cai WJ, Mei H, et al. Robust Decentralized Parameter Identification for Two-Input Two-Output Processes from Closed Loop Step Responses. Journal of Control, Engineering Practice. 2007;13:519.531.

6. Tumageanian A, Keyhani A. Identification of Synchronous Machine Linear Parameters From Standstill Step Voltage Input Data. IEEE Transactions on Energy Conversion. 1995;10(2):232-240.

7. Langley FJ. Testing Analog VLSI Circuits with Pulse Techniques. Proceedings of the International Test Conference. 1985:250-255.

8. Chin KR. Functional Testing of Circuits and SMD Boards with Limited Nodal Analysis. Proceedings of the International Test Conference. 1989:129-143.

9. Dai H, Souders TM. Time-Domain Testing Strategies and Fault Diagnosis for Analog Systems. IEEE Transactions on Instrumentation and Measurement. 1990;39(1):157-162.

10. Narahara K, Nakagawa S. Nonlinear traveling wave field effect transistors for amplification of short electrical pulses. IEICE Electronics Express. 2010;7(16):1188-1194. 